\title{
Lectura Del Diagrama De Tallo Y Hojas Por Estudiantes Chilenos De Educación Primaria
}

\author{
Reading The Stem And Leaf Diagram By Primary Education Chilean Students
}

\author{
Danilo Díaz-Levicoy* \\ Universidad Católica del Maule - (UCM) \\ Carmen Batanero** \\ Universidad de Granada - (UGR) \\ Pedro Arteaga ${ }^{* * *}$ \\ Universidad de Granada - (UGR) \\ Nuria Begué ${ }^{* * * *}$ \\ Universidad de Zaragoza - (UNIZAR)
}

\begin{abstract}
Resumen
El objetivo de este trabajo es describir las dificultades de los estudiantes chilenos de $6^{\circ}$ y $7^{\circ}$ curso de Educación Primaria (12-13 años) en la lectura del diagrama de tallo y hojas, y los niveles de lectura que logran. Para alcanzar este objetivo, se plantea a una muestra de 745 estudiantes de estos dos cursos y de diferentes ciudades chilenas una tarea abierta con dos preguntas basadas en la lectura de dicho diagrama. En la primera de ellas, se pide hallar el valor más frecuente y en la segunda calcular la frecuencia en un intervalo de valores. El análisis cualitativo de contenido de las respuestas permite categorizar las dificultades de lectura del diagrama y de comprensión de otros conceptos matemáticos. Los resultados muestran que un porcentaje bajo de estudiantes logran responder correctamente las preguntas planteadas. Entre los errores de lectura del gráfico destacan el no considerar las hojas o interpretarlas como cifras decimales de un número o como cifras de un valor entero. Se observan también confusión entre frecuencia y valor de la variable, máximo y moda e interpretación incorrecta de desigualdades. Por otro lado, solo una tercera parte de los estudiantes alcanza el nivel de lectura máximo permitido en la tarea. Estos resultados evidencian la dificultad para leer esta representación por parte los estudiantes de la muestra, no observándose diferencias significativas entre los dos cursos considerados.
\end{abstract}

Palabras clave: Diagrama de tallo y hojas, Comprensión, Nivel de lectura, Educación Primaria.

\footnotetext{
* Doctor en Ciencias de la Educación por la Universidad de Granada. Académico de la Facultad de Ciencias Básica, Universidad Católica del Maule (UCM), Talca, Chile. E-mail: dddiaz01@hotmail.com

** Doctora en Matemáticas por la Universidad de Granada. Catedrática de Didáctica de la Matemática en la Universidad de Granada (UGR), Granada, España. E-mail: batanero@ugr.es

*** Doctor en Didáctica de la Matemática por la Universidad de Granada. Profesor de Didáctica de la Matemática en la Universidad de Granada, Granada, España. E-mail: parteaga@ugr.es

${ }^{* * * *}$ Máster en Didáctica de la Matemática por la Universidad de Granada. Profesora de Didáctica de la Matemática en la Universidad de Zaragoza (UNIZAR), Zaragoza, España. E-mail: nbegue@correo.ugr.es
} 


\begin{abstract}
The aim of this research is to describe the difficulties and reading levels achieved by 6 th and 7 th grade Chilean primary education students in reading the stem and leaf plot. To achieve this objective, a sample of 745 students from these two courses and from different Chilean cities were given an open task with two questions based on the reading of this diagram. In the first question students should find the most frequent value and in the second compute the frequency in a range of values. The qualitative analysis of the answers serves to categorize the difficulties in reading the diagram and in understanding other mathematical concepts. The results show a low percentage of students who successfully answered the questions posed. The most frequent errors consist in not considering the leaf or in interpreting it as the decimal digits of a number or as the figures in an integer number. There was also confusion between frequency and value of the variable, maximum and mode, as well as incorrect interpretation of inequalities. On the other hand, only a third of students reached the maximum reading level allowed in the task. These results prove the difficulty of reading this representation by the students of the sample, were significant differences between the two school grades were not observed.
\end{abstract}

Keywords: Stem and leaf diagram, Understanding, Reading level, Primary Education.

\title{
1 Introducción
}

Los gráficos estadísticos son fundamentales para presentar y analizar la información sobre numerosos temas y tienen una fuerte presencia en los medios de comunicación y vida cotidiana y profesional. Su comprensión y correcta lectura son parte de la cultura estadística (Gal, 2002; Watson, 2013) y es una necesidad de la amplia disponibilidad de gráficos sobre datos de interés social, debido a la revolución tecnológica (Engel, 2019; Ridgway, 2016).

Esta relevancia ha llevado a incluir el aprendizaje de los gráficos estadísticos $\mathrm{y}$, en general, de la estadística en la Educación Primaria en muchos países. En el caso de Chile, país en que se realiza en estudio, las directrices curriculares del Ministerio de Educación (MINEDUC, 2012) hacen referencia explícita al trabajo con los siguientes tipos gráficos en el eje de aprendizaje titulado Datos y probabilidades: pictogramas (primero al cuarto curso), gráficos de barras (segundo al quinto curso), gráfico de puntos (tercer y sexto curso), gráficos de línea (quinto curso), diagramas de tallo y hojas (quinto y sexto curso) y gráficos de barra doble y sectores (sexto curso). Por otro lado, nuestro estudio previo de los libros de textos chilenos de matemática (Díaz-Levicoy, Batanero, Arteaga y Gea, 2016) permitió comprobar que estos cumplen las directrices curriculares, ya que contemplan todos estos gráficos en los niveles citados.

El diagrama de tallo y hojas se recomienda en las orientaciones curriculares chilenas, de acuerdo a los siguientes objetivos de aprendizaje:

- Quinto año. Utilizar diagramas de tallo y hojas para representar datos provenientes de 
muestras aleatorias (MINEDUC, 2013a, p. 43)

- Sexto año. Comparar distribuciones de dos grupos, provenientes de muestras aleatorias, usando diagramas de puntos y de tallo y hojas. (MINEDUC, 2013b, p. 43).

Sin embargo, este gráfico no suele trabajarse en la Educación Primaria en otros países; por ejemplo, no se menciona ni en las directrices curriculares ni en los libros de texto de Argentina (Díaz-Levicoy; Giacomone y Arteaga, 2017), Costa Rica (Jiménez-Castro, 2017), España (Díaz-Levicoy et al., 2016) o Guatemala y Venezuela (Salcedo, 2016).

Aunque crecen actualmente las investigaciones, que detallaremos en los antecedentes sobre la comprensión de los niños de diversos gráficos, no hemos encontrado estudios referidos al diagrama de tallo y hojas, tipo de gráfico en el que se centra el nuestro. Por este motivo, en esta investigación, nos planteamos como objetivo describir la capacidad y nivel de lectura que logran los estudiantes chilenos de $6^{\circ}$ y $7^{\circ}$ curso de Educación Primaria al trabajar con el diagrama de tallo y hojas. Dicha información permitirá a los maestros predecir y evaluar las posibles dificultades de sus estudiantes en estos niveles educativos.

\section{Fundamentos}

Nuestro trabajo se apoya en dos tipos de fundamentos. Por un lado, nos basamos en la categorización de niveles de lectura de un gráfico elaborada por Curcio y sus colaboradores (Curcio, 1989; Friel, Curcio y Bright, 2001). Por otro, en las investigaciones previas que han analizado la lectura de gráficos por parte de niños de edades similares a los que constituyen nuestra muestra.

\subsection{Niveles De Lectura De Los Gráficos Estadísticos}

La lectura de un gráfico estadístico es una actividad que puede tener diferentes niveles de dificultad, dependiendo de la pregunta que se formule en relación a dicha representación. Ello se debe a que un gráfico es un objeto semiótico complejo, formado por diferentes componentes, como las escalas, los datos representados, según un convenio que se debe conocer y la relación entre la representación y el contexto de la información. Como consecuencia, su lectura requiere una serie de procesos interpretativos de cada uno de estos componentes y del gráfico en su conjunto (Arteaga, Batanero, Contreras y Cañadas, 2012; Bertin, 1967). 
Dicha interpretación puede ser más o menos compleja, dependiendo de la pregunta que se haga sobre el gráfico, lo que da lugar a que varios autores hayan definido niveles de lectura. En este trabajo nos basamos en los definidos por Curcio y sus colaboradores (Curcio, 1989; Friel, Curcio y Bright, 2001), que son los siguientes y de los cuales solo evaluamos si los niños alcanzan los dos primeros:

- N1. Leer los datos. Cuando la pregunta planteada solo requiere la lectura literal de un elemento del gráfico estadístico. Por ejemplo, en el diagrama de tallo hojas se puede pedir leer uno de los datos, uniendo el número formado por el tallo y la hoja respectiva.

- N2. Leer dentro de los datos. Además de la lectura simple, la pregunta exige la comparación de datos del gráfico o la realización de cálculos aritméticos sencillos con los datos. Un ejemplo de este nivel sería buscar la moda de los datos representados en el diagrama.

- N3. Leer más allá de los datos. Conlleva deducir valores que no están representados en el gráfico. Por ejemplo, si los datos fuesen una serie de valores de venta en 10 meses consecutivos, pedir el valor esperado el mes número 11.

- N4. Leer detrás de los datos. Implica realizar una valoración crítica del gráfico, la forma de construirlo o de las conclusiones realizadas a partir del análisis del diagrama. Un ejemplo sería estudiar si el diagrama de tallo y hojas se puede utilizar para ciertos tipos de datos. Este nivel fue previamente descrito por Shaughnessy, Garfield y Greer (1996) y asumido posteriormente por Friel et al. (2001).

\section{Antecedentes}

En los últimos años, observamos un incremento de las investigaciones que analizan lectura de gráficos por parte de los niños, debido a la inclusión de los gráficos estadísticos en las directrices curriculares de Educación Primaria. A continuación detallamos las principales.

Guimarães (2002) estudia la interpretación de los gráficos de barras por 107 estudiantes de $3^{\circ}$ curso de Educación Primaria en Brasil, planteando tres actividades (dos con datos nominales y uno con datos ordinales). Los resultados muestran que el 72\% de los niños logran la lectura puntual (encontrar máximos, mínimos y localizar frecuencias o categorías) y 54,2\% de los estudiantes son capaces de realizar una extrapolación a un dato no representado en el gráfico, siendo más difícil leer una frecuencia no explícita. Se observan también fallos de comprensión variacional, cuando se pide localizar la parte del gráfico en que la variable 
estudiada tiene mayor aumento o disminución, tarea que sólo logran realizar el $26,3 \%$ de los niños.

Pagan, Leite, Magina y Cazorla (2008) estudian la lectura de tablas y gráficos estadísticos de 399 estudiantes (159 de $5^{\circ}$ y 80 de $8^{\circ}$ grado de Educación Primaria y 160 de $2^{\circ}$ de Educación Secundaria) en Brasil. Dos de sus ítems están relacionados con gráficos de barras. Sus datos muestran un logro del 67,3\%; obteniendo mejores resultados en las actividades que demandan un nivel N1 (84\% de respuestas correctas) frente al 43\% en las actividades que exigen un nivel $\mathrm{N} 2$ de lectura.

Canché (2009) aplica un cuestionario con actividades de opción múltiple sobre gráficos estadísticos (dos pictogramas, dos gráficos circulares, dos gráficos lineales, uno de barras) a una muestra de 206 estudiantes de $6^{\circ}$ curso en México. Los resultados, a nivel general, indican que el $69,1 \%$ responden correctamente a las actividades de nivel N1, 50,6\% en N2 y 47,5\% en N3. En los pictogramas, el 62,6\% logran el nivel N1, 45,6\% el N2 y 51\% el N3. En los líneas, el $41 \%$ logran el N1, 43\% el N2 y 26,7\% el N3. En los de sectores, 90,6\% el N1, 57,1\% el N2 y $63,4 \%$ el N3. Finalmente, la actividad donde interviene el gráfico de barras el $95,1 \%$ logran el N1, $62,6 \%$ el N2 y $50,5 \%$ el N3.

Fernandes y Morais (2011) analizan las respuestas de 108 estudiantes de $9^{\circ}$ grado a tres actividades relacionadas con la lectura de gráficos (diagrama de barras simples, gráfico circular y gráfico lineal). Obtienen $68 \%$ de respuestas correctas a las preguntas de nivel N1, 33\% a las de N3 y 24\% a las de N2. En el diagrama de barras, el 90\% alcanza el N1 y 23\% el N2. En el gráfico circular, el 96\% logran el N1, 31\% el N2 y 23\% el N3. En el gráfico de líneas el 19\% logra el N1, $14 \%$ el N2 y $43 \%$ el N3.

Fernandes, Santos y Pereira (2017) describen una secuencia de enseñanza centrada en el trabajo con tablas y gráficos estadísticos con 35 estudiantes de $5^{\circ}$ curso de Educación Primaria en Brasil. Analizan el aprendizaje comparando los resultados de un pre-test y un posttest. El 63,3\% contestaron correctamente la pregunta sobre un pictograma en el pre-test y un $100 \%$ en el post-test. El 86,7\% responde correctamente a un gráfico de barras en el pre-test y un $100 \%$ en el post-test en una pregunta de nivel $\mathrm{N} 1$ y $77,1 \%$ en el pre-test al $97,2 \%$ en el posttest a una pregunta de nivel N2.

Cruz (2013) analiza la lectura de gráficos estadísticos de 22 estudiantes de $3^{\circ}$ de Educación Primaria en Lisboa, dentro de un experimento de enseñanza, aplicando un pre test y un post test, que incluye dos actividades relacionadas con la lectura de gráficos (un pictograma y un gráfico de sectores). Los estudiantes alcanzan mejores resultados al trabajar con el 
pictograma (70\%) que con el gráfico de sectores (21,3\%). Respecto a los niveles de lectura, independiente del tipo de gráfico, responden correctamente el 53,8\% de las actividades N1, el $46,8 \%$ del N2 y $24,5 \%$ en las de N3.

Estas investigaciones se centran en los gráficos que usualmente incluyen las directrices curriculares de los países donde se han llevado a cabo cada investigación, pero no hemos encontrado estudios que se basen en diagramas de tallo y hojas, por lo que con este estudio aportamos información original sobre las dificultades de lectura de estos gráficos.

\section{Metodología}

Este estudio sigue una metodología de tipo cualitativa y descriptiva (Hernández, Fernández y Baptista, 2010), basada en el análisis de contenido de las respuestas escritas de los niños a las tareas planteadas. Dicho tipo de análisis se utiliza para el estudio sistemático de documentos escritos (Cohen, Manion y Morrison, 2011; López-Noguero, 2002).

En lo que sigue, describimos la muestra que formó parte del estudio, la tarea propuesta y detallamos el método de análisis de las respuestas.

\subsection{Muestra}

La muestra participante en el estudio estuvo formada por 745 estudiantes de Educación Primaria en Chile, 380 de $6^{\circ}$ curso (11-12 años) y 365 de $7^{\circ}$ curso (12-13años), pertenecientes a 13 centros educativos de siete ciudades diferentes de Chile. Estos estudiantes tenían una edad media de 12,3 años, siendo el 50,9\% mujeres y el $49,1 \%$ hombres. El acceso a las aulas se realizó previa autorización de los directores de los centros y de los profesores que atendían a los cursos, quienes colaboraron con el primer autor, que se desplazó a Chile para realizar la recogida de datos. La tarea se resolvió dentro de la clase de matemática y se explicó previamente a los niños el propósito del estudio, que era identificar sus posibles dificultades para mejorar la enseñanza del tema.

Los niños colaboraron con interés y, en una sesión posterior, se corrigió con ellos el ejercicio, para que la actividad tuviese un interés educativo para ellos. En los ejemplos mostrados en el artículo, asignamos a cada estudiante un código (Ex), donde $x$ corresponde a un número del 1 al 745 de acuerdo al orden de codificación de los datos de estos estudiantes. 


\subsection{Instrumento}

La tarea planteada a los estudiantes (Figura 1) se adaptó de un libro de texto de $6^{\circ}$ curso de Educación Primaria chileno (Ávila, Castro, Merino y Ramírez, 2013, p. 295). En él se plantean dos actividades de lectura de nivel N2 (leer dentro de los datos) en un diagrama de tallo y hojas, que representa una distribución de datos de las calificaciones de un grupo de estudiantes en una prueba. Se trata de un contexto familiar a los estudiantes, puesto que están acostumbrados a recibir calificaciones que contienen una parte entera y un número decimal. Dicho ítem fue sometido a juicio de expertos y pilotado antes de su aplicación definitiva.

En el siguiente gráfico se presentan las notas obtenidas (entre 2 y 7 ) por estudiantes de $6^{\circ}$ curso en una prueba de matemática (con una cifra decimal).

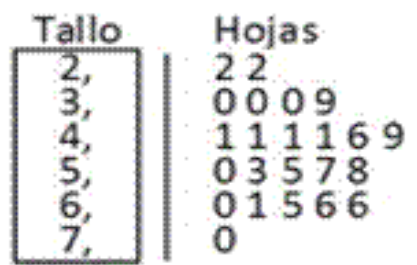

De acuerdo a esta información, responde:

1. ¿Qué nota es la que presenta mayor frecuencia?

2. ¿Cuántos estudiantes obtuvieron una nota mayor o igual a 5 ?

Figura 1: Ítem planteado a los estudiantes

Fuente: adaptado de Ávila et al. (2013, p. 295)

El diagrama de tallo y hojas fue introducido por Tukey (1977) y, como se observa en la Figura 1, representa todos los datos de un conjunto ordenados de menor a mayor. Cada dato se descompone en la parte entera (tallo) y decimal (hojas), representando cada tallo una única vez y tallos diferentes en distintas líneas. De modo que desde el gráfico se puede identificar directamente los datos: 2,2; 2,2; 3,0; 3,0; 3,0; 3,9; y así sucesivamente. Contando el número de hojas que corresponde a cada tallo, se puede obtener la frecuencia de cada tallo, o lo que es lo mismo, en un intervalo de valores. Por ejemplo, hay dos observaciones desde el 2 incluido hasta el 3 sin incluir. En resumen, en este gráfico, los valores se representan mediante la combinación de un tallo y una hoja, donde el tallo corresponde las primeras cifras (unidades, decenas, etc.) y las hojas indican la última unidad. Además, el número de hojas, en cada tallo, es la frecuencia de un intervalo (Tukey, 1977).

En el ejemplo propuesto, el tipo de datos (distribución) es de nivel 3 respecto al grado de complejidad semiótica definida por Arteaga (2011) y Batanero, Arteaga y Ruiz (2010), y es el mínimo nivel de complejidad de los datos que pueden ser representados en el diagrama de tallo y hojas, pues el nivel 1 sería simplemente un dato aislado o unos pocos datos aislados y el nivel 2 un listado de datos sin ordenar, mientras que, por definición en el diagrama de tallo y 
hojas los datos se presentan ordenados.

Para responder la primera pregunta, los estudiantes deben recordar los convenios de construcción del gráfico, reconocer que la columna tallo muestra la parte entera de las calificaciones y que cada número en la fila, asociado a cada tronco, corresponde a la parte decimal (hoja) de un número. Una vez comprendido esto, el estudiante debe identificar el valor que más se repite en el gráfico, que es el 4,1 y cuya frecuencia es 4 , sería la moda de la distribución.

Para responder la segunda pregunta, deben recordar el significado de la expresión igual y mayor y contar el número de hojas correspondientes a los tallos 5, 6 y 7, en total 11 valores. Puesto que las dos preguntas requieren realizar comparaciones entre subconjuntos de datos y la segunda también cálculo, corresponden a un nivel de lectura N2 (leer dentro de los datos) en la clasificación de Curcio y sus colaboradores.

\subsection{Método De Análisis}

Se realizó una revisión detallada y sistemática de las respuestas de los estudiantes a cada parte de la tarea, utilizando el análisis de contenido (Cohen, Manion y Morrison, 2011), considerado como una forma objetiva y sistemática de análisis, según los siguientes pasos:

- Se establecen, a priori, tres categorías para el análisis de las respuestas de los estudiantes (correctas, parcialmente correctas e incorrectas).

- En una segunda revisión se identifican los errores más comunes de las respuestas parcialmente correctas e incorrectas, depurando la clasificación mediante un proceso cíclico e inductivo.

- Un tercer análisis consiste en identificar el nivel de lectura alcanzado por cada alumno en cada pregunta $(\mathrm{N} 0, \mathrm{~N} 1, \mathrm{~N} 2)$, siguiendo los criterios previamente establecidos por Curcio y sus colaboradores, cuya aplicación se explicita en la sección 5.3.

- Finalmente se realiza un análisis descriptivo de los datos obtenidos.

\section{Resultados}

En este apartado describimos los resultados sobre la corrección de las respuestas y de los niveles de lectura alcanzados, finalizando con una síntesis de los resultados. 


\subsection{Respuestas A La Primera Pregunta: Nota De Mayor Frecuencia}

En la pregunta, sobre el valor de mayor frecuencia, hemos obtenido las siguientes categorías:

Respuesta correcta. Cuando leen correctamente el gráfico, identificando el valor de mayor frecuencia en el diagrama de tallo y hojas, lo que conlleva interpretar correctamente la parte entera (tallo) y decimal (hojas) que forman parte de las calificaciones. En consecuencia, el estudiante identifica el 4,1 como calificación modal, con frecuencia igual a 4. Un ejemplo de respuesta correcta es la de E16.

La nota 4,1 ya que se repite 4 veces (E16).

Respuesta parcialmente correcta. Cuando no se presentan errores conceptuales de tipo estadístico, llegando a reconocer el intervalo modal, pero no se determina la moda, debido a olvido parcial de los convenios de lectura del diagrama. Por ejemplo, se identifica correctamente el tallo que contiene la calificación más frecuente, pero se falla al explicitar la hoja o no se tiene en cuenta. Encontramos varias subcategorías:

- P1. No considera las hojas. Cuando el estudiante únicamente identifica el tallo que corresponde al intervalo donde se encuentra la calificación de mayor frecuencia, sin especificar la hoja, es decir, da el valor entero de la calificación sin su parte decimal. Estos estudiantes no llegan al primer nivel de lectura N1 en la interpretación del gráfico. En este caso tenemos dos posibles respuestas, aquellas en que dan como calificación modal 4 o las que asumen que el tallo representa la decena de un número y dan como calificación modal 40, como el caso siguiente:

La más frecuente fue el 40 (E50).

- P2. No diferencia la parte entera y la parte decimal en el diagrama. El estudiante asume que el tallo representa decenas y las hojas unidades, sin prestar atención a la parte decimal. Por ello, responden que la nota más frecuente es el 41, que no está dentro del rango de calificaciones usados en el sistema escolar chileno (de 1,0 a 7,0). En todo caso, se evidencia la capacidad de leer el gráfico al nivel N1 y comprende la idea de mayor frecuencia dentro de diagrama de tallo y hojas, como vemos a continuación:

La nota que representa mayor frecuencia es el 41 (E57).

- P3. Interpreta las hojas como la parte decimal de un mismo número. Cuando se 
interpreta el valor del tallo como parte entera y las hojas respectivas como cifras de la parte decimal de un único número. El estudiante no comprende el diagrama y no es capaz de leerlo, pues reconoce la fila de mayor frecuencia, pero no interpreta correctamente el significado de las hojas, como en la respuesta de E166.

La nota que presenta mayor frecuencia es 4,111169 (E166).

- P4. Omite el valor del tallo e interpreta las hojas como un solo número. Es una variante de la respuesta anterior, pero ahora omiten la parte entera y responden con un número formado por todas las hojas del tallo 4 , sin considerar que cada hoja representa una calificación diferente. Estas respuestas muestran que han leído incorrectamente el diagrama, pues aunque encuentra la fila de mayor frecuencia, no recuerdan el convenio que diferencia el tallo y las hojas. Como ejemplo vemos la respuesta de E321.

Es 111169 (E321).

Respuesta incorrecta. Corresponden a las respuestas en las que se observan errores conceptuales en elementos estadísticos requeridos para la solución, lo que lleva a una réplica incorrecta o sin sentido en el contexto dado. En esta categoría hemos encontrado las siguientes categorías:

- I1. Confunde moda y máximo. Cuando proporciona la calificación máxima (7,0o 70) en lugar del valor de mayor frecuente, es decir, están confunden el máximo con la moda e implícitamente la idea de frecuencia y valor de la variable. Este es un error que aparece en Cobo (2003) en su trabajo sobre comprensión de las medidas de tendencia central. Ejemplo de esta situación es la respuesta de E3 que, además, no diferencia entre la parte entera y decimal del número, aunque ha realizado una lectura literal del diagrama.

70 es la mayor (E3).

- I2. Confunde moda y mínimo. Cuando contestan con la calificación mínima de la distribución $(2,2)$, lo cual puede indicar confusión por la forma en que se presenta el tallo del diagrama de tallo y hojas, donde el valor mínimo está en la parte más alta. En estas respuestas también hay confusión conceptual (COBO, 2003) y, además, se considera solo la parte entera o se toman todas las hojas como la parte decimal del número, como se observa a continuación:

2,22 (E495).

- I3. Suma de las hojas del tallo de mayor frecuencia. Cuando se responde 5 (tallo), 
identificando las hojas con la frecuencia de valores, y la frase mayor frecuencia como suma de las hojas de cada tallo, por lo que la lectura de la gráfica también es incorrecta. Ejemplo de ello es la respuesta de E398, quien señala que la frecuencia es 23, que se obtiene de sumar las hojas del tallo $5(0+3+5+7+8)$. De nuevo aparece la confusión entre frecuencia y valor de la variable descrita por Cobo (2003).

E1 5 porque tiene 23 estudiantes (E398).

- I4. Hoja de mayor frecuencia. Cuando se considera la hoja de mayor frecuencia o el valor de la misma, sin tener en cuenta el valor de tallo. Pueden indicar el 0, que corresponde al valor que se repite más veces entre las hojas, o el 6 que es su frecuencia. Esto evidencia que los estudiantes no recuerdan los convenios de lectura del diagrama de tallo y hojas y no llegan a comprender el valor de los datos representados.

Un cero (E266).

- I5. Responde con un intervalo de valores. Cuando indican que la calificación modal está incluida en un intervalo, confundiendo los conceptos de moda y valor modal y dando el límite inferior 4 o 40. Por ejemplo, E534 sitúa la moda entre los valores 40 y 49, interpretando los tallos como decenas y las hojas como unidades. Esta respuesta, al menos, supone una lectura literal del gráfico.

La que representa mayor frecuencia es de 40 hasta 49 (E534).

- I6. Otros errores. Incluimos las respuestas que no se han descrito anteriormente y que no tienen sentido aparente, o que se presentan en menor frecuencia. Por ejemplo, E35 no es capaz de identificar un valor modal.

Ninguna ya [que] todas son diferentes (E35).

En la Tabla 1 presentamos la distribución de los tipos de respuestas según su corrección. Globalmente, cerca del 80\% de los estudiantes resolvió la actividad, y en su mayoría en forma parcialmente correcta $(31,2 \%)$. Los estudiantes de $6^{\circ}$ curso presentan un mayor nivel de éxito con casi un $10 \%$ de diferencia en respuestas correctas con los de $7^{\circ}$, y un menor porcentaje de preguntas en blanco.

Tabla 1: Porcentaje de estudiantes según corrección de la respuesta en la primera pregunta

\begin{tabular}{lccc}
\hline Tipo de respuesta & $\begin{array}{c}\mathbf{6}^{\mathbf{0}} \text { curso } \\
(\mathrm{n}=380)\end{array}$ & $\begin{array}{c}\mathbf{7}^{\mathbf{0}} \text { curso } \\
(\mathrm{n}=365)\end{array}$ & $\begin{array}{c}\text { Total } \\
(\mathrm{n}=745)\end{array}$ \\
\hline Correcta & 31,1 & 21,4 & 26,3
\end{tabular}




\begin{tabular}{lccc}
\hline \multicolumn{1}{c}{ Tipo de respuesta } & $\begin{array}{c}\mathbf{6}^{\mathbf{0}} \text { curso } \\
(\mathrm{n}=380)\end{array}$ & $\begin{array}{c}\mathbf{7}^{\mathbf{0}} \text { curso } \\
(\mathrm{n}=365)\end{array}$ & $\begin{array}{c}\text { Total } \\
(\mathrm{n}=745)\end{array}$ \\
\hline Parcialmente correcta & 32,2 & 30,3 & 31,2 \\
Incorrecta & 21,8 & 19,2 & 20,5 \\
No contesta & 15 & 29 & 21,9 \\
\hline
\end{tabular}

Fuente: elaborado por los autores

El porcentaje de errores encontrados en las respuestas parcialmente correctas (Tabla 2) es, en general, pequeño, salvo en el consistente en no tener en cuenta las hojas, donde los estudiantes identifican la fila que contiene a la moda, sin interpretar correctamente el significado de la hoja en el diagrama.

Tabla 2: Porcentaje de estudiantes con diferentes tipos de errores en la primera pregunta

\begin{tabular}{lccc}
\hline \multicolumn{1}{c}{ Tipo de error } & $\begin{array}{c}\mathbf{6}^{\mathbf{0}} \text { curso } \\
(\mathrm{n}=380)\end{array}$ & $\begin{array}{c}\mathbf{7}^{\mathbf{0}} \text { curso } \\
(\mathrm{n}=365)\end{array}$ & $\begin{array}{c}\text { Total } \\
(\mathrm{n}=745)\end{array}$ \\
\hline P1. No considera las hojas & 27,4 & 26 & 26,7 \\
P2. No diferencia la parte entera y la parte decimal en el diagrama & 1,1 & 0,8 & 0,9 \\
P3. Interpreta las hojas como la parte decimal de un mismo número & 3,2 & 3 & 3,1 \\
P4. Omite el valor del tallo e interpreta las hojas como un solo número & 0,5 & 0,5 & 0,5 \\
I1. Confunde moda y máximo & 5,5 & 4,7 & 5,1 \\
I2. Confunde moda y mínimo & 1,3 & 3 & 2,1 \\
I3. Suma de las hojas del tallo de mayor frecuencia & 4,2 & 6 & 5,1 \\
I4. Hoja de máxima frecuencia & 3,4 & 2,5 & 3 \\
I5. Responde con un intervalo de valores & 0,8 & 0,3 & 0,5 \\
I6. Otros errores & 6,6 & 2,7 & 4,7 \\
\hline
\end{tabular}

Fuente: elaborado por los autores

\subsection{Segunda Pregunta: Frecuencia De Calificación 5 O Mayor}

A continuación se describen las respuestas a la segunda pregunta, en que los estudiantes deben calcular la frecuencia de notas superiores o iguales a 5.

Respuesta correcta. Cuando localizan en el diagrama todos los valores que cumplen en la condición (valores con tallo 5, 6 y 7) y suman el número de hojas que corresponde a cada uno (11), como en la solución de E713:

11 estudiantes. Porque 5 personas se sacaron un $5 \mathrm{y}$ tanto, 5 personas se sacaron un $6 \mathrm{y}$ tanto, 1 persona un 7 (E713).

Respuestas parcialmente correctas. Cuando no se presentan errores conceptuales de tipo estadístico, pero se observan dificultades debidas a olvido parcial de los convenios de lectura del diagrama. En lo que sigue mostraremos las subcategorías encontradas, que repiten algunas encontradas en la primera pregunta. 
- P1. Interpreta las hojas de cada tallo como cifras decimales de un mismo número. Cuando los estudiantes interpretan cada tallo como la parte entera de un único número, cuya parte decimal son el conjunto de hojas asociados y entienden que la frecuencia asociada a cada tallo es la unidad. En consecuencia, los estudiantes indican como respuesta 3 o 2, dependiendo si consideran o no el tallo de valor 5. Por ejemplo:

3 personas obtuvieron uno nota igual o mayor a $5(5,6$ y 7$)$ (E567).

- P2. Interpreta las hojas asociadas a un tallo como parte de un número entero, omitiendo el tallo. En este caso la respuesta no es consistente con la pregunta planteada, como ocurre con E307, que escribe el número formado por las hojas del tallo 4, que no puede corresponder al número de estudiante, al ser mucho mayor que el total.

111169 (E307).

- P3. Suma las hojas de los tallos iguales a 6 o mayor. Cuando responden con la suma de algunos valores de los tallos o las hojas del diagrama, como en la respuesta de E246, que se muestra a continuación, y que no se relaciona con la pregunta planteada:

Obtuvieron 23 estudiantes igual a la nota 5 y 18 estudiantes obtuvieron mayor nota (E246).

Respuesta incorrecta. Cuando se evidencia dificultad en el manejo de desigualdades, aunque en muchos casos el estudiante conoce los convenios de lectura del diagrama. Se pueden clasificar en la forma siguiente:

- I1. Interpretar la desigualdad en forma estricta, excluyendo el valor 5. Cuando se interpreta la expresión mayor o igual como mayor, considerando sólo las calificaciones superiores a 5 y excluyendo el valor 5. Este error no se debe atribuir al desconocimiento de la lectura del diagrama, que se hace correctamente, sino a dificultades en la comprensión de desigualdades, como ocurre con E124.

Los estudiantes que obtuvieron una nota mayor o igual que 5 fueron 10 (E124)

- I2. Interpretar la desigualdad en forma estricta, excluyendo todos los valores menores a 6. Es una variante de la respuesta anterior, pero en esta ocasión se excluyen todos los valores cuya parte entera es 5, por lo que calcula sólo con las calificaciones comprendidas entre 6,0 y 7,0, como en el ejemplo de E44:

6 estudiantes obtuvieron una nota mayor o igual a 5 (E44).

- I3. Confundir igualdad y desigualdad. Cuando consideran solo las hojas del tallo 5, por 
lo que su respuesta puede ser 4 o 5, dependiendo si considera o no el valor exacto 5,0, también atribuible a dificultades con las desigualdades, como el siguiente ejemplo:

Los estudiantes que obtuvieron mayor o igual a 5 son 5 estudiantes (E106).

- I4. Otros errores. Cuando presentan valores que no tiene un sentido aparente y/o que son poco frecuentes. Ejemplo de esta situación es la de E45, que da como valor del número de alumnos un número decimal.

$5,3(\mathrm{E} 45)$.

En la Tabla 3 analizamos la corrección a la respuesta al cálculo de la frecuencia mayor igual a un valor, respondida por cerca del 75\% de la muestra, predominando las respuestas correctas $(29,3 \%)$, que, junto con las parcialmente correctas alcanzan más del $50 \%$ de los estudiantes. Además, los estudiantes de $6^{\circ}$ curso presentan mayor proporción de respuestas correctas e incorrectas y menor porcentaje de respuestas en blanco que los de $7^{\circ}$ curso.

Tabla 3: Porcentaje de estudiantes según tipo de respuesta en la segunda pregunta

\begin{tabular}{cccc}
\hline Tipo de respuesta & $\begin{array}{c}\mathbf{6}^{\mathbf{0}} \text { curso } \\
(\mathrm{n}=380)\end{array}$ & $\begin{array}{c}\mathbf{7}^{\mathbf{0}} \text { curso } \\
(\mathrm{n}=365)\end{array}$ & $\begin{array}{c}\text { Total } \\
(\mathrm{n}=745)\end{array}$ \\
\hline Correcta & 31,6 & 26,8 & 29,3 \\
Parcialmente & 26,1 & 22,7 & 24,4 \\
Incorrecta & 26,3 & 14 & 20,2 \\
No contesta & 16,1 & 36,4 & 26 \\
\hline
\end{tabular}

Fuente: elaborado por los autores

En la Tabla 4 mostramos los porcentajes de los diferentes errores encontrados en las respuestas parcialmente correctas e incorrectas, generalmente pequeños. Una parte de estos errores se deben a la interpretación de desigualdades y no a la lectura del diagrama y aparecen en total (categorías I1 a I3) en el 18,2\% de los estudiantes de $6^{\circ}$ curso y en el 9,6\% de los de $7^{\circ}$ curso, donde ha disminuido esta dificultad, que en todo caso tiene poca incidencia. Mayor proporción presentan $\left(26,1 \%\right.$ en $6^{\circ}$ curso y $22,7 \%$ en $7^{\circ}$ curso) las respuestas incorrectas que se deben a no recordar los convenios de lectura del gráfico, siendo el error más frecuente realizar cálculos con tallos u hojas o interpretar las hojas de cada tallo como cifras decimales de un único número. 
Tabla 4: Porcentaje de estudiantes con diferentes tipos de errores en la segunda pregunta

\begin{tabular}{|c|c|c|c|}
\hline Tipo de error & $\begin{array}{l}\mathbf{6}^{\mathbf{0}} \text { curso } \\
(\mathrm{n}=380)\end{array}$ & $\begin{array}{l}7^{\mathbf{0}} \text { curso } \\
(\mathrm{n}=365)\end{array}$ & $\begin{array}{c}\text { Total } \\
(\mathrm{n}=745)\end{array}$ \\
\hline $\begin{array}{l}\text { P1. Interpretar las hojas de cada tallo como cifras decimales de un mismo } \\
\text { número }\end{array}$ & 8,2 & 6 & 7,1 \\
\hline $\begin{array}{l}\text { P2. Interpreta las hojas como la parte decimal de un sólo número y da una } \\
\text { respuesta sin sentido }\end{array}$ & 6,1 & 6,3 & 6,2 \\
\hline P3. Suma las hojas de los tallos iguales a 6 o mayor & 11,8 & 10,4 & 11,1 \\
\hline I1. Interpretar la desigualdad en forma estricta, excluyendo el valor 5 & 1,1 & 1,4 & 1,2 \\
\hline $\begin{array}{l}\text { I2. Interpretar la desigualdad en forma estricta, excluyendo todos los valores } \\
\text { menores a } 6 .\end{array}$ & 2,6 & 2,2 & 2,4 \\
\hline I3. Confundir igualdad y desigualdad. & 14,5 & 6 & 10,3 \\
\hline I4. Otros errores & 8,2 & 4,4 & 6,3 \\
\hline
\end{tabular}

Fuente: elaborado por los autores

\subsection{Nivel De Lectura}

Además de analizar la corrección y tipos de error en las respuestas, se estudió el nivel de lectura alcanzado (Curcio, 1989; Friel et al., 2001) en cada pregunta. Los criterios para establecer este nivel fueron los siguientes:

- NO. Corresponde por un lado a los estudiantes que no responden las preguntas y por otro los que no llegan a leer correctamente el diagrama. Por tanto se incluyen en este nivel todos los errores descritos anteriormente consistentes en no considerar las hojas o no considerar el tallo, es decir, las respuestas consideradas como incorrectas. Ejemplo de este nivel es la respuesta de E32 a la segunda pregunta, donde no llega a una lectura literal del diagrama.

Los estudiantes que obtuvieron una nota (E32).

- N1. Leer los datos. Cuando realizan una lectura literal de un valor en el diagrama de tallo y hojas, pero cometen errores al realizar cálculos o comparaciones con los datos. Por ejemplo, E3 ha leído el valor 70 en el gráfico, pero dicho valor no es el de más frecuencia, por tanto, no ha sido capaz de comparar las frecuencias de diferentes datos en la primera pregunta.

70 es la mayor (E3).

- N2. Leer dentro de los datos. Cuando, además de leer correctamente algún dato del gráfico, son capaces de comparar algunos valores para el cálculo de la moda en la primera pregunta o realizar cálculos sencillos, para hallar la frecuencia correspondiente a un intervalo en la segunda. Por ejemplo, E51 demuestra haber leído correctamente los 
datos y ha identificado el dato de mayor frecuencia en la primera pregunta e igualmente E713 lee correctamente el diagrama y encuentra la frecuencia pedida en la segunda pregunta:

La nota que tiene más frecuencia es el 4,1 (E51).

11 estudiantes. Porque 5 personas se sacaron un $5 \mathrm{y}$ tanto, 5 personas se sacaron un $6 \mathrm{y}$ tanto, 1 persona un 7 (E713).

En la Tabla 5 presentamos el porcentaje de estudiante que alcanzan los diferentes niveles de lecturas en cada pregunta. En ella se observa que apenas una tercera parte o menos lleva al nivel leer dentro de los datos (N2), donde además de la lectura literal se ponen en juego procesos matemáticos sencillos y/o comparación de valores.

Son menos aún los que llegan al nivel N1, es decir, al menos leen correctamente el gráfico y la mayoría no lo alcanza, quedando a nivel N0, debido a los diferentes errores de interpretación del gráfico que se han descrito al analizar las respuestas.

Al comparar por curso, los estudiantes de $6^{\circ}$ alcanzan mejor nivel que sus compañeros en la primera pregunta y algo menos en la segunda, debido en este caso, no a la lectura del gráfico, sino a no haber interpretado correctamente las desigualdades, lo que les ha llevado a no realizar correctamente los cálculos requeridos.

Tabla 5: Porcentaje de niveles de lectura en las actividades propuestas

\begin{tabular}{ccccccc}
\hline & \multicolumn{3}{c}{ Primera pregunta } & \multicolumn{3}{c}{ Segunda pregunta } \\
\hline Nivel de lectura & $\begin{array}{c}\mathbf{6}^{\mathbf{0}} \text { curso } \\
(\mathrm{n}=380)\end{array}$ & $\begin{array}{c}\mathbf{7}^{\mathbf{0}} \text { curso } \\
(\mathrm{n}=365)\end{array}$ & $\begin{array}{c}\text { Total } \\
(\mathrm{n}=745)\end{array}$ & $\begin{array}{c}\mathbf{6}^{\mathbf{0}} \text { curso } \\
(\mathrm{n}=380)\end{array}$ & $\begin{array}{c}\mathbf{7}^{\mathbf{0}} \text { curso } \\
(\mathrm{n}=365)\end{array}$ & $\begin{array}{c}\text { Total } \\
(\mathrm{n}=745)\end{array}$ \\
\hline 2 & 31,1 & 21,4 & 26,3 & 31,6 & 26,8 & 29,3 \\
1 & 7,4 & 5,8 & 6,5 & 18,2 & 9,6 & 13,9 \\
0 & 61,5 & 72,8 & 67,2 & 50,4 & 63,5 & 56,7 \\
\hline
\end{tabular}

Fuente: elaborado por los autores

\section{Discusión Y Conclusión}

La incorporación de los gráficos estadísticos en las directrices curriculares actuales del MINEDUC (2012) plantea desafíos importantes al profesorado y a los autores de los libros de texto, dado que tienen incidencia directa con la formación de los niños de Educación Primaria. En este sentido, la finalidad de este trabajo fue proporcionar información sobre la comprensión del diagrama de tallo y hojas por parte de los estudiantes.

Los resultados obtenidos en nuestra muestra fueron pobres, pues las respuestas correctas a la primera pregunta son sólo el $26,3 \%$ en la primera pregunta y el $29,3 \%$ a la segunda. Estos 
resultados mejoran si también consideramos las parcialmente correctas, donde los estudiantes no presentan errores conceptuales importantes, pero fallan en reconocer los convenios de lectura del gráfico, llegando a un 57,5\% en la primera pregunta y un 53,7\% en la segunda. En todo caso el porcentaje de respuestas incorrectas o en blanco es muy alto en los dos grupos, $42,4 \%$ en la primera y $46,3 \%$ en la segunda pregunta.

Observamos que estos resultados son peores que los obtenidos en investigaciones centradas en otros tipos de gráfico. Así, Pagan et al. (2008) obtiene 67,3\% de respuestas correctas por niños de $5^{\circ}$ de Educación Primaria en Brasil en la lectura de gráficos de barras y Fernandes et al. (2017) 63,3\% en el gráfico de líneas y 86,7\% en el de barras antes de su experiencia de enseñanza.

De nuestros antecedentes, solo son similares a los obtenidos por estudiantes de $3^{\circ}$ de Educación Primaria en Cruz (2013) al trabajar con un gráfico de sectores (21,3\%), situación que cambia al trabajar con un pictograma (70\% de éxito). Concluimos entonces la mayor dificultad que implica la lectura del diagrama de tallo y hojas en relación a los gráficos propuestos en otras investigaciones.

Al analizar el nivel de lectura que logran las respuestas de los estudiantes, vemos que el nivel máximo exigido en la primera actividad es alcanzado únicamente por el $26,3 \%$ de los participantes y por el 29,3\% en la segunda, no llegando al nivel N1 en la mayoría de los casos. En otras investigaciones sobre lectura de gráficos el nivel de lectura N2 se ha alcanzado por el $43 \%$ de los estudiantes en los gráficos de barras (Pagan et al., 2008), 45,6\% en los pictogramas y 57,1\% en los de sectores (Canché, 2009) y 46,8\% en la investigación de Cruz sobre pictogramas y gráficos de sectores. Este resultado refuerza nuestra interpretación de la mayor dificultad del diagrama de tallo y hojas en comparación con los anteriores gráficos estadísticos.

Nuestro trabajo se complementa con una descripción detallada de los errores que cometen los estudiantes en la lectura del gráfico, que principalmente se explican por no recordar los convenios de representación del mismo. Aunque la interpretación del tallo en el diagrama es más sencilla, una dificultad particular aparece en la lectura de las hojas, que se toman como cifras del número cuyo primer dígito es el tronco correspondiente, bien interpretándolo como un número entero o como la parte decimal de un número decimal.

Se añaden otras dificultades no estrictamente debidas al gráfico, como la confusión entre frecuencia y valor de la variable, o la confusión entre máximo y moda, así como la interpretación de desigualdades.

En resumen, nuestros resultados sugieren que podría ser conveniente reflexionar sobre 
el lugar que ocupa el diagrama de tallo y hojas en el currículo chileno, analizando la conveniencia de retrasar su estudio un par de años como se viene realizando en otros países.

\section{Agradecimientos}

Trabajo realizado con apoyo del Proyecto EDU2016-74848-P (AEI, FEDER) y Grupo FQM126 (Junta de Andalucía).

\section{Referencias}

Arteaga, P. (2011). Evaluación de conocimientos sobre gráficos estadísticos y conocimientos didácticos de futuros profesores (Tesis Doctoral). Universidad de Granada, España.

Arteaga, P., Batanero, C., Contreras, J. M. y Cañadas G. (2012). Understanding statistical graphs: A research survey. BEIO. Boletín de Estadística e Investigación Operativa, 28(3), 261-277.

Ávila, J., Castro, C., Merino, R. y Ramírez, P. (2013). Matemática 6 ${ }^{\circ}$ Básico. Tomo II. Santiago: Santillana.

Batanero, C., Arteaga, P. y Ruiz, B. (2010). Análisis de la complejidad semiótica de los gráficos producidos por futuros profesores de educación primaria en una tarea de comparación de dos variables estadísticas. Enseñanza de las Ciencias, 28(1), 141-154.

Bertin, J. (1967). Semiologie graphique. Paris: Gauthier-Villars.

Canché, L. (2009). La comprensión gráfica de los alumnos del nivel primaria (Tesis de Máster). Universidad Autónoma de Yucatán, México.

Cobo, B. (2003). Significados de las medidas de posición central para los estudiantes de secundaria (Tesis Doctoral). Universidad de Granada, España.

Cohen, L., Manion, L. y Morrison, K. (2011). Research methods in education. Londres: Routledge.

Cruz, A. (2013). Erros e dificuldades de alunos de $1 .{ }^{\circ}$ ciclo na representação de dados estatísticos (Tesis de Máster). Universidad de Lisboa, Portugal.

Curcio, F. R. (1989). Developing graph comprehension. Reston, VA: NCTM.

Díaz-Levicoy, D., Batanero, C., Arteaga, P. y Gea, M.M. (2016).Gráficos estadísticos en libros de texto de Educación Primaria: Un estudio comparativo entre y Chile. BOLEMA. Boletim de Educação Matemática, 30(55), 713-737.

Díaz-Levicoy, D., Giacomone, B. y Arteaga, P. (2017). Caracterización de los gráfícos 
estadísticos en libros de texto argentinos del segundo ciclo de Educación Primaria. Profesorado. Revista de Currículum y Formación del Profesorado, 21(2), 299-326.

Engel, J. (2019). Statistical literacy and society. En J. M. Contreras, M. M. Gea, M. M. LópezMartín y E. Molina-Portillo (Eds.), Actas del Tercer Congreso International Virtual de Educación Estadística (pp. 1-17). Granada: Grupo de Investigación de Educación Estadística de la Universidad de Granada.

Fernandes, J. A. y Morais, P. C. (2011). Leitura e interpretação de gráficos estatísticos por alunos do $9^{\circ}$ ano de escolaridade. Educação Matemática Pesquisa, 13(1), 95-115.

Fernandes, R., Santos, G. y Pereira, R. (2017). Ensino e aprendizagem de gráficos e tabelas nos anos iniciais de escolarização. UNIÓN. Revista Iberoamericana de Educación Matemática, 50, 41-61.

Friel, S., Curcio, F. y Bright, G. (2001). Making sense of graphs: critical factors influencing comprehension and instructional implications. Journal for Research in mathematics Education, 32(2), 124-158.

Gal, I. (2002). Adult's statistical literacy: Meaning, components, responsibilities. International Statistical Review, 70(1), 1-25.

Guimarães, G. (2002). Interpretando e construindo gráficos de barras (Tesis doctoral). Universidad Federal de Pernambuco, Brasil.

Hernández, R., Fernández, C. y Baptista, P. (2010). Metodología de la investigación. México: McGraw Hill.

Jiménez-Castro, M. (2017). Los gráficos estadísticos en el currículo y los libros de texto de Educación Primaria en Costa Rica (Trabajo Fin de Máster). Universidad de Granada, España.

López-Noguero, F. (2002). El análisis de contenido como método de investigación. XXI. Revista de Educación, 4, 167-180.

MINEDUC (2012). Matemática Educación Básica. Bases curriculares. Santiago: Unidad de Currículum y Evaluación.

MINEDUC (2013a). Matemática. Programa de estudio quinto año básico. Santiago: Unidad de Currículum y Evaluación.

MINEDUC (2013b). Matemática. Programa de estudio sexto año básico. Santiago: Unidad de Currículum y Evaluación.

Pagan, A., Leite, A. P., Magina, S. y Cazorla, I. (2008). A leitura e interpretação de gráficos e tabelas no Ensino Fundamental e Médio. En V. Gitirana, F. Bellemain y V. Andrade (Eds.), Anais do $2^{\circ}$ Simpósio Internacional de Pesquisa em Educação Matemática (pp. 1-10). Recife: Universidad Federal de Pernambuco.

Ridgway, J. (2016). Implications of the data revolution for statistics education. International 
Statistical Review, 84(3), 528-549.

Salcedo, A. (2016). Gráficos estadísticos en libros de texto para Educación Primaria de Guatemala y Venezuela. Educação Matemática Pesquisa, 18(3), 1141-1163.

Shaughnessy, J. M., Garfield, J. y Greer, B. (1996). Data handling. En A. J. Bishop, K. Clements, C. Keitel, J. Kilpatrick y C. Laborde (Eds.), International handbook of mathematics education (pp. 205-237). Dordrecht, The Netherlands: Kluwer Academic Publishers.

Tukey, J. (1977). Exploratory data analysis. Reading, MA: Addison-Wesley.

Watson, J. M. (2013). Statistical literacy at school: Growth and goals. Mahwah, NJ: Lawrence Erlbaum.

Submetido em: 26/03/2019

Aceito em: 09/09/2019 